\title{
SEXUALIDADE HUMANA: NÍVEL DE CONHECIMENTO DOS ACADÊMICOS DE ENFERMAGEM
}

Human Sexuality:

Knowledge Level of the Nursing Students

Sexualidad Humana:

Nivel de Conocimiento de los Estudiantes de Enfermería

Lincoln Vitor Santos

Aline de Oliveira Ribeiro
Maria Pontes de Aguiar Campos

Maria Cláudia Tavares de Mattos

\section{Resumo}

Trata de uma pesquisa quali-quantitativa descritiva, cujo objetivo foi verificar como o acadêmico de Enfermagem conceitua termos básicos para o entendimento do tema Sexualidade Humana. Constatou-se que o estudante possui um nível regular de conhecimentos relativos ao tema, pois, embora consiga atribuir conceitos corretos a termos mais simples, quando Ihe são solicitadas definições mais detalhadas, em patamar mais profundo e que pedem uma base teórica concreta, o estudante se confunde e não consegue alcançar êxito. Tal situação exige uma reavaliação quanto à necessidade da inclusão de conteúdos curriculares sobre sexualidade, evitando o ocultamento e a invisibilidade da mesma.

Palavras-chave: Estudantes de Enfermagem. Sexualidade. Educação em Enfermagem.

\section{Abstract}

The present study is a qualitative and quantitative research which purpose was verifies how nursing undergraduate conceptualizes essential terms to comprehension of Human Sexuality. It was detected that student has a regular level of knowledge relatives to theme, because, although he attributes right concepts to simple terms, when details definitions are ask him, he confused himself and he can't success. This situation requires a revaluation of the necessity of inclusion of contents about sexuality, avoiding its covertness and invisibility.

\section{Resumen}

El presente estudio es una investigación cualitativa y cuantitativa descriptiva, cuyo objetivo fue verificar como el estudiante de Enfermería conceptúa términos básicos para el entendimiento del tema Sexualidad Humana. Se constató que el estudiante tiene un nivel regular de conocimientos relativos al tema, pues, aunque logre atribuir conceptos correctos a termos más sencillos, cuando le solicitan definiciones más detalladas, en un nivel más profundo y que piden una base teórica concreta, el estudiante se confunde y no logra éxito. Éste hecho exige una nueva evaluación de la necesidad de la inclusión de contenidos curriculares sobre sexualidad, evitando su ocultamiento y su invisibilidad.
Keywords:

Students, Nursing. Sexuality. Education, Nursing.
Palabras clave: Estudiantes de Enfermería. Sexualidad. Educación en Enfermería. 


\section{INTRODUÇÃO}

A universidade vem enfatizando, cada vez mais, a importância de o cliente ser atendido de forma holística, devendo ser percebido como indivíduo. Faz-se, portanto, necessário que o planejamento de sua Assistência de Enfermagem seja singular e inclua 0 aconselhamento na esfera da sexualidade.

No passado, a Enfermagem era exercida por pessoas que acreditavam prestar um serviço a Deus, e, com o advento da Enfermagem moderna, Florence Nightingale propôs um padrão de comportamento às enfermeiras, no qual a sexualidade era suprimida.

A Enfermagem tem a permissão social de tocar qualquer parte do corpo do outro para atender às suas necessidades humanas básicas e prestar cuidados, mas os enfermeiros agem como assexuados. Desta forma, têm dificuldade de interagir terapeuticamente com a sexualidade do outro e com a sua própria, dessexualiza o corpo do cliente e transforma-o em objeto, ignorando aspectos da sexualidade do cliente, limitandose à limpeza e higienização dos órgãos genitais ${ }^{1,2}$.

Tudo o que remete à sexualidade é algo desconhecido e produtor de ansiedade para a maioria das pessoas. Para 0 acadêmico, Sexualidade está intimamente relacionada à Enfermagem, pois trata-se de uma profissão que tem de lidar com o corpo do outro. No entanto, durante a graduação, a sexualidade é pouco discutida, levando o aluno ao embaraço quando tem de vivenciar questões relacionadas ao tema ${ }^{3}$.

A sexualidade humana e a sua relação com a Enfermagem conta com escassa literatura nacional; logo, debater o tema em trabalhos científicos permitirá que se compreendam melhor as deficiências apresentadas na graduação de Enfermagem, no que tange ao tema, e a qualidade da assistência prestada ${ }^{1}$.

0 interesse por este estudo foi despertado ao detectarse que a maior parte dos alunos se sentiu constrangida e despreparada ao lidar com a sexualidade dos clientes sob seus cuidados, durante as aulas práticas das disciplinas Introdução à Enfermagem, Enfermagem Cirúrgica, Enfermagem Médica e Enfermagem Psiquiátrica.

Portanto, traçou-se como objetivo verificar como o(a) acadêmico(a) de Enfermagem conceitua sexualidade humana, atividade sexual, disfunção sexual e homossexualidade.

Pretende-se com este trabalho contribuir para que acadêmicos e professores possam, juntos, identificar maneiras de inserir na formação do Enfermeiro debates e explanaç̧̃̃es relacionadas à sexualidade do cliente, repensando uma formação em que tais questões permanecem obscurecidas.

\section{CAMINHO METODOLÓGICO}

Optou-se por uma pesquisa com abordagem qualiquantitativa, de natureza descritiva, desenvolvida na cidade de Aracaju, Estado de Sergipe, Brasil, no Campus da Saúde Professor João Cardoso Nascimento Júnior, da Universidade Federal de Sergipe (UFS).

A população-alvo da pesquisa constituiu-se de todos os 106 (cento e seis) acadêmicos do curso Enfermagem Bacharelado, da UFS, que estavam do quinto ao nono período curricular. A limitação a esses períodos fez-se necessária, uma vez que representam o ciclo profissional da graduação, no qual os alunos entram em contato com os pacientes prestando cuidados de enfermagem.

A amostra de 32 alunos foi selecionada pelo critério probabilístico aleatório, a partir de uma lista única, organizada em ordem alfabética, independente do período curricular, numerada seqüencialmente do número 1 até 106. Os sujeitos foram selecionados por sorteio aleatório a partir do número correspondente da lista única, até a quantidade pretendida, que corresponde a $30,2 \%$ da população-alvo.

Os nomes dos participantes foram trocados por um código composto, na ordem, por um número arábico (ordem de sorteio), uma letra (sexo) e outro número arábico (período curricular), para preservação do anonimato dos mesmos.

Este estudo foi submetido à análise do Comitê de Ética e Pesquisa do Hospital Universitário da UFS, e aprovado por atender a Resolução 196/96.

Os sujeitos do estudo que foram sorteados e que concordaram em participar da pesquisa assinaram o Termo de Consentimento Livre e Esclarecido (TCLE).

Aplicou-se um questionário composto de 5 perguntas fechadas, 4 abertas e 1 de múltipla escolha, os quais foram distribuídos nas salas de aula do Campus da Saúde da UFS e respondidos individualmente, sem a liberdade de consulta a fontes bibliográficas.

Utilizaram-se as técnicas de análise estatística e de conteúdo para esta pesquisa. Foi feita uma leitura flutuante das respostas e, então, as mesmas foram distribuídas em categorias, em correspondência aos objetivos da pesquisa, para serem analisadas quanto ao conteúdo e ao número de vezes em que apareceram.

Durante a seleção, os dados foram minuciosamente examinados e submetidos à verificação crítica, para que falhas e informações confusas, distorcidas e incompletas pudessem ser detectadas e sanadas.

A codificação permitiu a categorização dos dados relacionados entre si. Em um primeiro momento, os dados foram classificados sob categorias, e depois cada categoria recebeu uma denominação.

Pela tabulação, foi possível verificar com mais facilidade a inter-relação entre os dados coletados. A compreensão e a interpretação foram mais rápidas com a representação gráfica. A análise estatística foi feita pela evidência das relações entre o fenômeno estudado e seus fatores causais.

\section{RESULTADOS E DISCUSSÃO}

Foram questionados os conhecimentos que os acadêmicos de Enfermagem têm sobre aspectos fundamentais da Sexualidade, tais como Sexualidade Humana, Atividade Sexual, Dificuldade Sexual e Homossexualidade.

Estes termos foram selecionados por serem essenciais para o entendimento da complexidade que é o tema Sexualidade Humana, visto que a literatura ${ }^{4}$ preconiza como metas para a educação sexual a desmistificação do tema, o entendimento da anatomia e fisiologia sexuais, incluindo o detalhamento da conduta sexual entre duas pessoas. 
Em relação ao sexo, houve um predomínio $(78,13 \%)$ do feminino. Referente à idade, temos $87,50 \%$ na faixa etária dos 20 aos 25 anos de idade, 9,38\% dos 26 aos 30 anos e $3,13 \%$ dos 36 aos 40 anos.

Com relação ao período curricular que estavam cursando à época da entrevista, a distribuição apresentou-se da seguinte maneira: $15,63 \%$ (5) no $5^{\circ}$ período, $21,88 \%$ (7) no $6^{\circ}$ período, $21,88 \%$ (7) no $7^{\circ}$ período, $25,00 \%$ (8) no $8^{\circ}$ período e $15,63 \%$ (5) no $9^{\circ}$ período

Com relação ao conceito de Sexualidade Humana, 100,00\% (32) dos sujeitos responderam corretamente ao escolherem a assertiva sexualidade é o conjunto de caracteres especiais, fisiológicos ou morfológicos, internos ou externos, determinados a partir de uma integração de fatores biológicos, psicológicos, sócio-econômicos, culturais, étnicos, espirituais e religiosos ${ }^{5,6}$.

Este resultado demonstra o quão instigante e envolvente é o tema tratado, uma vez que leva o aluno a buscar conhecimentos sobre 0 assunto, isto porque a sexualidade é inseparável dos demais aspectos da vida por ser uma necessidade humana básica que funciona como um complemento da identidade do ser humano, manifestando-se na saúde, na liberdade de expressão e no pensamento ${ }^{5,6}$.

E ainda porque a sexualidade é uma das formas do indivíduo interagir com o mundo, de se comportar, é pulsão de vida inerente a todo ser em todos os momentos da sua vida pessoal ou profissional, pública ou privada, sadia ou doente ${ }^{6}$ e consiste numa construção social, datada e culturalmente localizada, transcendendo a genitalidade e a simples reprodução da espécie ${ }^{7}$.

Este tema não vem sendo tratado de forma sistemática na graduação em Enfermagem, o que forma profissionais de saúde com dificuldades e inabilidade em lidar com o tema, pois durante a graduação, e até a pós-graduação, pouca ou nenhuma informação Ihes foi dada. Conflitos emocionais, angústias, sentimentos de culpa e repressões estão ligados a esta ignorância do profissional no que concerne à sexualidade, inclusive à sua própria8 .

No que diz respeito ao conceito que os sujeitos atribuíram ao termo Homossexualidade, 3,13\% da amostra conceituou de forma inapropriada, ou seja, considerou-o uma manifestação patológica, conferindo à homossexualidade uma doença psicológica.

Por longo tempo, a homossexualidade foi encarada como uma manifestação patológica. Muitos psicólogos, psiquiatras e psicanalistas defenderam a idéia de que o homossexual seria alguém que não amadureceu completamente e por isso seria incapaz de se relacionar com o sexo oposto.

Essa idéia foi responsável pela suposição de que essas pessoas eram doentes e passíveis de cura 9 . Entretanto, o Conselho Federal de Psicologia estabeleceu uma resolução que promete punir os profissionais que se prestam a curar a homossexualidade ${ }^{10}$.

Diferentemente, 96,88\% da amostra atribuíram conceito concordante com a maioria dos especialistas que consideram a homossexualidade a atração sexual entre pessoas do mesmo sexo, que em geral leva ao orgasmo ${ }^{11,12}$, o que demonstra, mais uma vez, que, devido à relevância do tema atribuída pelo aluno, esse buscou um conhecimento mais ampliado.

Quando se investigou o conceito de Atividade Sexual, a maioria, ou seja, 78,13\% (25), respondeu corretamente, em consonância com autores ao conceituar atividade sexual como um termo geral que inclui atos como beijos, carícias, cópula, masturbação, penetração anal e sexo oral ${ }^{12}$. Acrescentam a esse conceito os afagos, as bolinações e 0 tribadismo entre mulheres e dizem que a atividade sexual tem como fim o prazer e o orgasmo ${ }^{13}$.

No entanto, $21,88 \%$ (7), apesar de terem conceituado corretamente o termo Sexualidade Humana, genitalizaram 0 Sexo, conceituando-o como ato sexual, esquecendo que dessa forma limitam as possibilidades de se obter prazer.

Embora o aluno tenha buscado enriquecer seus conhecimentos, tal achado poderia não ter sido encontrado se a academia incluísse em seus programas curriculares a abordagem mais apropriada desse assunto.

Ato sexual, por sua vez, é a união dos genitais propriamente dita, considerado, pela maioria das pessoas, como o fim sexual normal. Esse termo possui como sinônimos a cópula e o coito. 0 sexo anal é outra forma de ato sexual, consistindo na penetração do pênis no ânus, prática muito comum entre os homossexuais e em uso crescente em casais heterossexuais ${ }^{14,15}$.

Sobre a conceituação do termo Dificuldade Sexual, a maior parte dos sujeitos, isto é, 65,63\% (21), o fizeram de modo errado ao dizer que se trata de uma situação em que a pessoa não consegue lidar com a sexualidade, seja falando, seja praticando 0 ato sexual, pois não foi devidamente esclarecida sobre 0 assunto. Em suma, consideraram como a dificuldade diante de assuntos e situações que envolvam a sexualidade.

Justifica-se esse percentual relativamente alto de erros, devido à utilização do termo Dificuldade em substituição a Disfunção, recomendado pela literatura ${ }^{16}$, pois essa entende que o termo Dificuldade Sexual remete à idéia de uma situação passageira e passível de cura, desde que trabalhada adequadamente, ao contrário de Disfunção, que tem um caráter mais estigmatizador.

Apenas 34,38\% (11) o conceituaram corretamente como distúrbios em que 0 indivíduo apresenta respostas sexuais insuficientes e falta de desejo, com um funcionamento incompleto da atividade sexual e insatisfação da relação sexual, sem atingir 0 orgasmo ${ }^{16}$.

Esses resultados demonstram o quanto o conhecimento do acadêmico de Enfermagem é insuficiente, pois, quando se tenta discutir mais profundamente o tema, o que requer uma base teórica sólida, percebe-se que o aluno não consegue alcançar o conceito adequado, necessitando, portanto, que esta abordagem seja contemplada nos cursos superiores de Enfermagem.

\section{CONSIDERAÇÕES FINAIS}

Sobre o conhecimento da população estudada referente à sexualidade, todos os sujeitos $(100,00 \%)$ entrevistados conceituaram corretamente a Sexualidade Humana.

Quanto à definição de Atividade Sexual, 78,13\% dos entrevistados responderam adequadamente, entendendo que o ser humano não limita as relações sexuais à penetração e pode buscar infinitas formas de prazer. No entanto, 21,88\% dos entrevistados têm uma visão limitada da atividade sexual como algo exclusivamente ligado à união dos genitais. 
Ao conceituar Homossexualidade, $96,88 \%$ o fizeram de forma acertada. Um sujeito $(3,12 \%)$ atribuiu uma manifestação patológica à homossexualidade.

No que se refere ao conceito de Dificuldade Sexual, $34,38 \%$ da amostra estudada a definiram corretamente. Porém, $65,63 \%$ dos sujeitos erraram ao associar este termo à situação em que a pessoa lida com dificuldade com assuntos e fatos que envolvem a sexualidade.

Constatou-se, com esta pesquisa, que o acadêmico de Enfermagem possui um nível regular de conhecimentos relativos ao tema Sexualidade Humana, pois, embora consiga atribuir conceitos corretos a termos mais simples, quando the são solicitadas definições mais detalhadas, em patamar mais profundo e que exija uma base teórica concreta, o estudante se confunde e não consegue alcançar êxito.

0 assunto é tratado de forma limitada e inadequada durante a graduação em Enfermagem, aparecendo superficialmente e em poucas disciplinas do tronco profissional. 0 Enfermeiro recebe uma formação na qual há a idéia de que o paciente é assexuado, pois, enquanto todas as suas necessidades de proteção, segurança, conforto, oxigenação, nutrição,

\section{Referências}

1. Pelá NTR, Melo AS, Santana WMS, Nhamba AL. A sexualidade humana no contexto da assistência de enfermagem. Rev Bras Sex Hum 1995 jan; 6 (1): 99-113.

2. Pupulim JSL, Sawada NO. 0 cuidado de enfermagem e a invasão da privacidade do doente: uma questão ético-moral. Rev Latino-am Enfermagem 2002 maio/jun; 10 (3): 433-38.

3. Pereira AL. Enfermeira/o não tem sexo (?) Representação social de graduandas/os de enfermagem sobre sexualidade. [dissertação de mestrado] Rio de Janeiro (RJ): Escola de Enfermagem/UERJ; 2000.

4. Garschagen DM, et al, editores. Enciclopédia Barsa. São Paulo (SP): Melhoramentos; 2000. Sexologia. v. 14, p. 258-60

5. Potter PA, Perry AG. Grande tratado de enfermagem prática: clínica e prática hospitalar. Tradução de Hildegard Thiemann Buckup e Terezinha Oppido]. $3^{\text {a }}$ ed. São Paulo(SP): Santos Liv Ed, 2002: 280-87; 621-75.

6. Sena SA. Orientação sexual nas escolas públicas: mudanças ocorridas nos alunos [monografia]. Aracaju (SE): Departamento de Enfermagem e Nutrição/Centro de Ciências Biológicas e da Saúde/UFS; 2004.

7. Toniette M. Sexualidade... ou sexualidades? Bol Inf CEPCos, 2004 mar; 10:3.

8. Lopes GP, Nascimento LG, Rezende WC. Os profissionais da saúde e a educação sexual. Rev Bras Sex Hum 1992 jan/jun; 3 (1): 23-6.

9. Garschagen DM, et al, editores. Enciclopédia Barsa. São Paulo (SP): Melhoramentos; 2000. Homossexualismo. v. 9, p. 109-10.

10. Rigoletto RN. Mais uma vez sobre a suposta cura da homossexualidade. Bol Inform CEPCos, 2004 jan; 10:1.

11. Quintella A, Dieterich DHM. Sexualidade. São Paulo (SP): Saraiva; 1992.

12. Goldenson RM, Anderson KN. Dicionário de sexo. $3^{\text {a }}$ ed. São Paulo (SP): Ática; 1992.

13. Laino E, Fonseca MCC, Rodrigues Júnior OM. Atitudes de estudantes universitários para com a homossexualidade masculina e a AIDS. Rev Bras Sex Hum 1990 jul/dez; 1 (2): 90-8. higienização, excreção e hidroeletrolíticas são amplamente debatidas, o tema sexualidade, quando aparece, o faz em poucas disciplinas e o profissional deixa de considerá-la quando presta a Assistência de Enfermagem.

Conhecer a sexualidade faz com que o indivíduo compreenda o seu eu interior e social, sendo papel do profissional de Enfermagem ajudar os clientes a manter e aumentar os conhecimentos nessa área. Contudo, a falta de informações, padrões éticos conflitantes, crenças religiosas, ansiedade e sentimentos de culpa do profissional podem interferir negativamente na sua atuação ao lidar com as questões da sexualidade.

Tal situação exige uma (re)avaliação quanto à necessidade de inclusão urgente de conteúdos sobre sexualidade, já que a manutenção do ocultamento e da invisibilidade da sexualidade limita a criatividade, a sensibilidade e a liberdade de expressão do Enfermeiro ${ }^{17}$.

Espera-se que, com a compreensão e a apreensão dos resultados deste trabalho, possa haver uma reformulação na grade curricular do curso de Enfermagem no que concerne ao tema em questão, no qual este assunto possa ser discutido, despido de tabus e preconceitos.

14. Cunha JA, organizador. Dicionário de termos de psicanálise de Freud. $10^{\mathrm{a}}$ ed. Rio de Janeiro (RJ): Globo; 1998.

15. Guyton AC, Hall JE. Tratado de fisiologia médica. $10^{\mathrm{a}}$ ed. Rio de Janeiro (RJ): Guanabara Koogan.; 2002: 857-82.

16. Faria CM. Sexualidade e auto-estima. Bol Inform CEPCos, 2003 ago; 9:8.

17. Ressel LB. Vivenciando a sexualidade na assistência de enfermagem: um estudo na perspectiva cultural. [tese de doutorado] São Paulo (SP): Escola de Enfermagem/USP; 2003.

\section{Sobre os Autores}

\section{Lincoln Vitor Santos}

Enfermeiro Assistencial do Programa de Saúde da Família em Itabaiana/ SE. Docente do Curso de Técnico em Enfermagem em Aracaju/SE.

\section{Maria Pontes de Aguiar Campos}

Enfermeira, Doutoranda em Saúde da Criança e do Adolescente pela FMRP/USP. Docente do Departamento de Enfermagem e Nutrição da Universidade Federal de Sergipe (UFS).

\section{Aline de Oliveira Ribeiro}

Enfermeira Assistencial do Programa de Saúde da Família em Aquidabã/ SE. Docente do Curso de Técnico em Enfermagem em Aracaju/SE.

\section{Maria Cláudia Tavares de Mattos}

Doutoranda em Saúde da Criança e do Adolescente pela FMRP/USP. Docente do Departamento de Enfermagem e Nutrição da UFS. 